\title{
Structure, Properties, and Degradation of Ultrathin Ionomer Films in Catalytic Layers of Fuel Cells
}

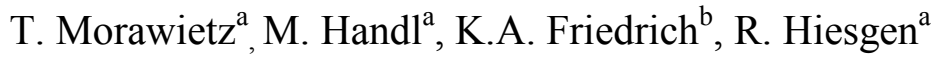 \\ ${ }^{a}$ University of Applied Sciences Esslingen, Kanalstr. 33, 73728 Esslingen, Germany \\ ${ }^{\mathrm{b}}$ Institute of Engineering Thermodynamics, German Aerospace Center, \\ Pfaffenwaldring 38-40, 70569Stuttgart, Germany
}

The structure of electrodes for proton-exchange membrane fuel cells and electrolysers has an important impact on performance and degradation. Ionomer films covering $\mathrm{Pt} / \mathrm{C}$ agglomerates in fuel cell electrodes have a thickness in the range of 4 to $15 \mathrm{~nm}$ and differ from the bulk structure with their mostly lamellar films. The formation of such nanothin films deposited by drop-casting and self-assembly was studied by atomic force microscopy. For different equivalent weights and different solvents of Aquivion ${ }^{\circledR}$ and Nafion ${ }^{\circledR}$ dispersions, the size and structure of the dispersion particles, of sub-monolayers, single and multilayers was investigated on graphene, platinum, mica, and silicon. The size of dispersion particles strongly depends on the solvent. On hydrophobic graphene, the height of ionomer bundles and layers measures $1.5 \mathrm{~nm}$, significantly less than the $2.5 \mathrm{~nm}$ height found on hydrophilic $\mathrm{Pt}, \mathrm{Si}$, and mica.

\section{Introduction}

Fuel cell electrodes are typically porous compounds of few nanometer-sized catalyst particles deposited on mesoporous carbon with diameters of 10-30 nm, mixed with an ionically conducting binder, mostly Nafion or Aquivion ionomers are used; nevertheless also electrode designs without ionomer using water as ionically conducting phase were also successfully applied (1-3). The carbon particles form agglomerates that are (partly) covered by ionomer and have an average size in the range of $100 \mathrm{~nm}$ as reported in ref. (4). The performance and duration of polymer electrolyte fuel cells depend on all components, although, the nano-structured electrodes have a large impact. The detailed nanostructure of the electrodes determines the macroscopic properties of the membraneelectrode-assembly (MEA) and thereby performance and degradation. It was reported that, i.e. the ionomer fraction and the kind of solvent, which have an impact on the structure of the ionomer layers, influence the MEA properties (5-7). For example, the average specific conductivity of the electrodes decreased with smaller film thickness (8). Furthermore, it was reported that for differently prepared MEAs the irreversible degradation rate was smaller for MEAs with initially thinner ionomer layers (4). The ionomer coverage of the agglomerates depends on the local surface energy of the carbon and thereby also on Pt loading (9).

Since the reduction of platinum loading as precious metal with limited resources and high prizes is an issue, its impact on the fuel cell performance was studied. A reduced Pt loading leads to an increase of surface energy with an impact on the ionomer coverage 
(9). Below a Pt loading of $0.15 \mathrm{mg}^{\cdot} \mathrm{cm}^{-2}$, an increasing transport resistance was found. The analysis performed by i.e. Mathias et al. concluded that the transport of oxygen through the Nafion layers is the major cause (10). Recently, an alternative explanation was provided by Muzaffar et al. stating that water layers covering the catalyst agglomerates are the main reason for the additional transport resistance (11). In this analysis the authors assume a partly ionomer coverage of the Pt-carbon agglomerates. In this case oxygen penetrates the agglomerate mainly through the gas/water interface without necessity to permeate through the ionomer.

Since the detailed structure of the electrodes is of importance, an analysis of their nanostructure was performed $(4,12)$. The ionomer phase is very sensitive to varying humidity and temperature and reacts with immediate dimension changes. An meaningful analysis of the electrodes needs to be performed at controlled humidity and temperature close to operational conditions to ensure the significance of measurements for fuel cell operation (13). Shrinkage/extension leads not only to changes in film thicknesses but also to a different ionomer coverage of the agglomerates $(4,12)$. In this respect as suitable analysis method atomic force microscopy (AFM) was used in this study. With materialsensitive AFM tapping mode, a high contrast between the ionomer- and the $\mathrm{Pt} / \mathrm{C}$ phase, i.e. adhesion force, deformation or stiffness mapping allows to study the distribution and size of the ionomer phase (Figure 1a) (4,13). High-resolution AFM adhesion mapping that exhibits the ionomer as bright phase showed separated ionomer films wrapped around the partly ionomer covered agglomerates with few embedded ionomer clusters (Figure 1b). The ionomer layers range in size from 4 to $15 \mathrm{~nm}$. After fuel cell operation, the thickness further decreased caused by chemical degradation (4,14). From i.e. modeling studies it was concluded that these nanothin films have a minimum thickness of $4 \mathrm{~nm}$ and represent only one ionomer backbone layer that covers a water film at the hydrophilic surface or two backbone bilayers with a sandwiched water film, depending on the substrate surface energy $(15,16)$. The nanothin films in the electrodes were found to be mostly lamellar, deduced from the linear swelling of the ionomer films with humidity increase $(15,16)$. For thicker ionomer films and low water content modelling shows that the formation of inverted micelles instead of layers becomes more advantageous $(15,16)$. It is well-known that the properties of such thin films differ strongly from that of the bulk ionomer, for example in conductivity as reported i.e. by Paul et al. from the analysis of self-assembled Nafion films on different substrates. The thickness of these self-assembled films was mainly dependent on the ionomer concentration with a minimum of $4 \mathrm{~nm}(1,8)$. A review of the properties of ionomer in PEMC electrodes can be found in ref. (1); a general review on ionomers can be found in ref. (18).

In a typical ink for fuel cell electrode preparation, i.e. for air brush spraying, doktorblade, or screen printing deposition, the concentration of ionomer is in the range of $0.1-4 \%$, respectively. Depending on the ionomer concentration and local surface energy which is determined by, i.e the fraction, size, and type of Pt and carbon, a formation of sub-monolayers or closed ionomer layers by self-assembling is expected. For this reason, the initial stages of formation of such nanothin films on model surfaces were studied. 


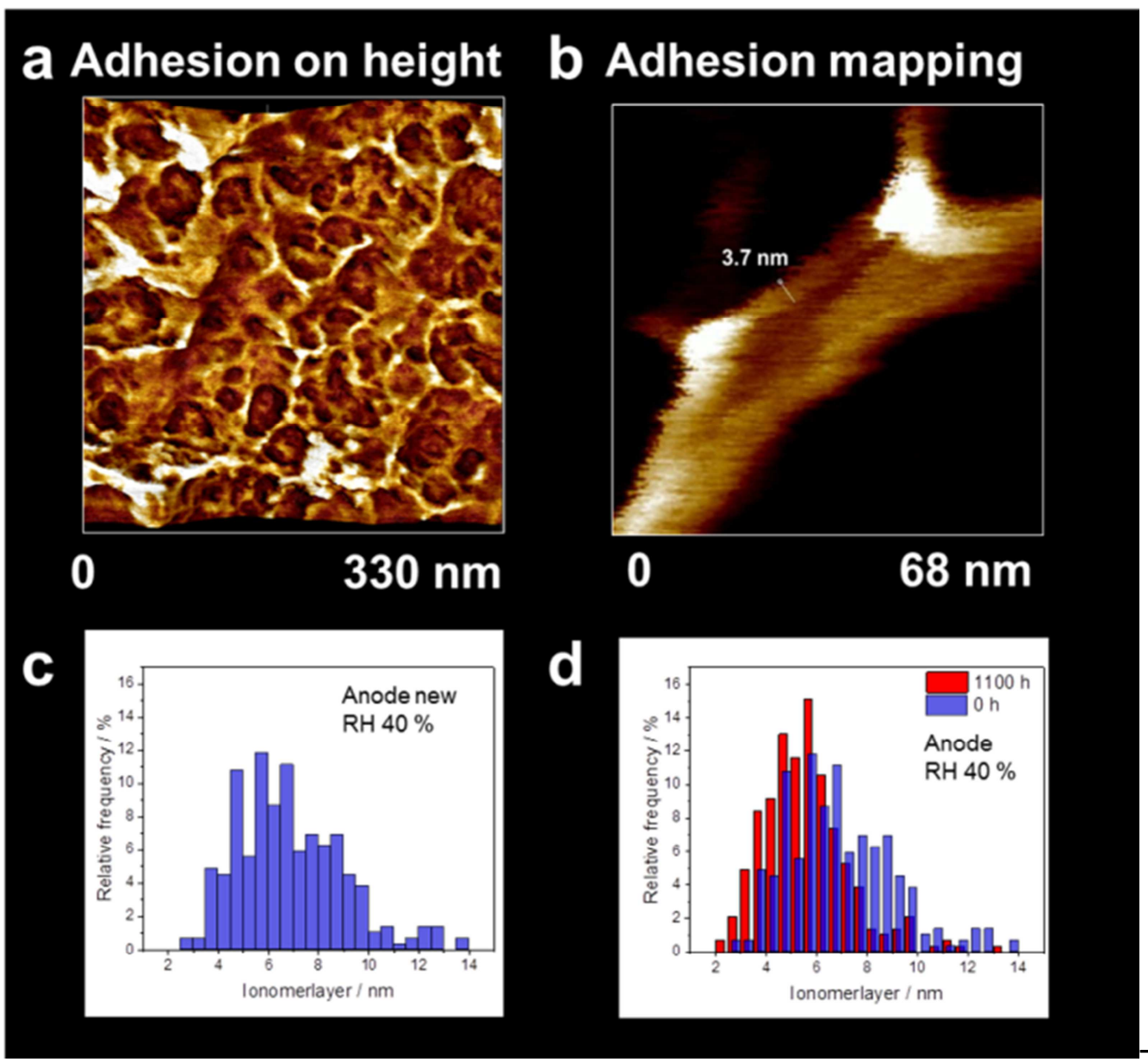

Figure 1. (a) AFM adhesion mapping of an electrode cross-section, bright color indicates ionomer, (b) high-resolution adhesion mapping of ionomer layers between agglomerates, (c) distribution of Aquivion film thicknesses at $40 \% \mathrm{RH}$ at a new anode cross-section, and (d) distribution of film thickness at new (blue) and $1100 \mathrm{~h}$ operated (red) anode cross-sections; the lengths refer to the total image size.

\section{Dispersion Particles}

The size and agglomeration of PFSA has been studied mainly for Nafion dispersions (19-23). Most authors report cylindrical aggregates or bundles. Their size and shape depends on the solvent and the equivalent weight of the ionomer. Ghelichi and Eikerling reported from a general modelling approach including a variety of different ionomer architectures that the particle size depends on the detailed molecular structure and the properties of the solvent (24). The bundles have a dense hexagonal packing consisting of ionomer backbones, the sulfonic acid groups are oriented to the sides. With increasing hydrophobicity the bundles became larger, while an increased density of sulfonated sidechains lead to a decrease in size. Small coulombic interaction leads to a decrease, strong interaction to an increase in bundle size. Welch et al. reported on Nafion particle sizes in pure and varying fractions of water/2-propanol solvents (21). They found well-defined cylindrical particles with a size of approximately $15 \mathrm{~nm}$ x $4.6 \mathrm{~nm}$ in glycerol, large 200 $\mathrm{nm}$ aggregates in water/isopropanol mixtures, and a random-coil conformation in $\mathrm{N}$ - 
methylpyrrolidone. Large secondary clusters of 200-400 nm were also measured in isopropanol/alcohol (IPA) by Ngo et al. (5). The Nafion molecules formed primary aggregated particles at medium IPA fractions, in dilute IPA/water solutions within the range of 20-45 wt.\%, these primary aggregated particles formed secondary ionic clusters via inter-sulfonate ionic aggregations. The primary aggregated particles changed from rod-like to coil-like structure above an IPA concentration of $70 \mathrm{wt} . \%$. From the included TEM images the bundle sizes were estimated in a range of 10 to $10^{2} \mathrm{~nm}$.

In order to get a first insight into the film formation in electrodes, model experiments using drop-cast deposition of ionomer from dispersion were performed. The size of the particles in dispersion, the first stages of sub-monolayer coverage to monolayer coverage, and the formation of closed films were studied with AFM. As hydrophilic and hydrophobic substrates including the materials relevant for electrodes, mica, Si, a Pt(110) single crystal, a Pt sputter layer, and graphene sheets detached from highly oriented pyrolytic graphite (HOPG) crystal were chosen.

\section{Experimental}

The AFM measurements were performed with a Bruker Multimode 8 . The materialsensitive evaluation delivers, i.e. adhesion force, stiffness, and deformation. Details can be found in ref. (25-27). For ionomer deposition, water-based and hydro-alcoholic dispersions of Nafion ${ }^{\circledR} 1100$ with an equivalent weight $(\mathrm{EW})$ of $(1100 \pm 20) \mathrm{g} \cdot \mathrm{mol}^{-1} \mathrm{SO}_{3} \mathrm{H}$, and different grades of Aquivion ${ }^{\circledR}$ PFSA were used: D83-25BS $(830 \pm 20) \mathrm{g} \cdot \mathrm{mol}^{-1} \mathrm{SO}_{3} \mathrm{H}$, water based) and D79-25BS (790 \pm 20$) \mathrm{g} \cdot \mathrm{mol}^{-1} \mathrm{SO}_{3} \mathrm{H}$ (water-based).

a
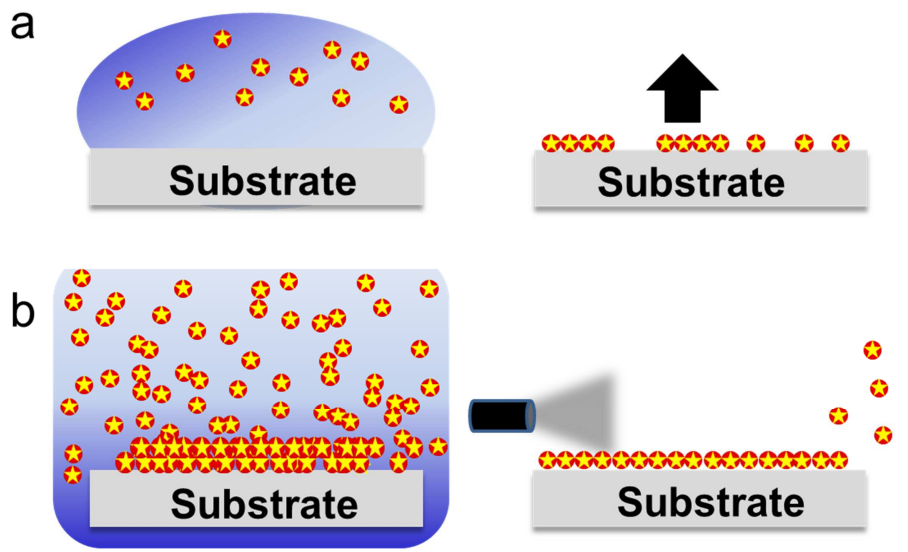

Figure 2. (a) Experimental procedure for sub-monolayer preparation by drop casting from ionomer concentration of 0.0025-0.005 wt.\%, evaporation time $1 \mathrm{~h}$ for water-based and $1 \mathrm{~min}$ for alcoholic dispersions, (b) film formation by self-assembling from dispersion with $0.25 \mathrm{wt} . \%$ ionomer and subsequent blowing off the residual dispersion.

For size determination of the dispersion particles and sub-monolayer films with AFM, a drop with an ionomer concentration between 0.0025 wt. $\%$ and 0.005 wt. $\%$ of waterbased or hydro-alcoholic ionomer dispersion was placed on the substrates ("dropcasting") and dried at room temperature (RT); in case of water-based dispersion for $1 \mathrm{~h}$ 
and $1 \mathrm{~min}$ for hydro-alcoholic dispersions (Figure 2a). Nanothin films were prepared by self-assembling for $2 \mathrm{~h}$ in $0.25 \mathrm{wt} . \%$ ionomer dispersion with subsequent blowing off the residual solvent with argon similar to the procedure described by Paul et al. (Figure 2b) without further curing (28). For comparison of the ionomer coverage on $\mathrm{Pt}$ and $\mathrm{Si}$ substrate self-assembled on the same sample, the Pt sputter layer was partly removed from the Si substrate using a razor blade.

Before ionomer deposition, the surfaces of all substrates were examined by AFM on their roughness and cleanliness. The AFM determined $R_{a}$ roughness values are given in brackets. A freshly flame-annealed $\operatorname{Pt}(110)$ crystal $\left(\mathrm{R}_{\mathrm{a}}=0.2 \mathrm{~nm}\right)$, a Pt-sputter film on $\mathrm{Si}$ $\left(\mathrm{R}_{\mathrm{a}}=0.8 \mathrm{~nm}\right)$, cleaned by a mixture of 3 parts of $\mathrm{H}_{2} \mathrm{SO}_{4}$ acid and 1 part of $30 \mathrm{vol} \%$ of $\mathrm{H}_{2} \mathrm{O}_{2}$ solution, a $\mathrm{Si}$ wafer $\left(\mathrm{R}_{\mathrm{a}}=0.1 \mathrm{~nm}\right)$, freshly cleaved mica sheets $\left(\mathrm{R}_{\mathrm{a}}=0.044 \mathrm{~nm}\right)$, and graphene sheets, freshly detached from a highly oriented pyrolytic graphite crystal $\left(\mathrm{R}_{\mathrm{a}}=0.023 \mathrm{~nm}\right)$, were used as substrates.
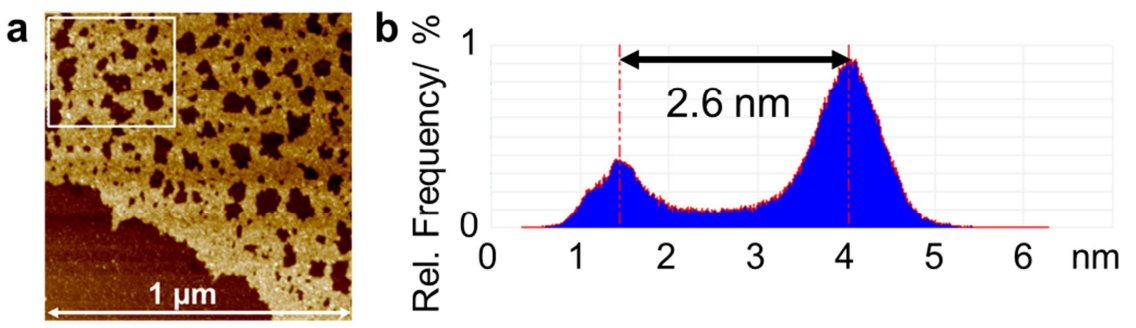

Figure 3. (a) AFM height image of drop-cast water-based Aquivion D79-25BS film from 0.005 wt.\% ionomer dispersion on mica with selected area for determination of film thickness, (b) distribution of height values.

As depicted in Figure 3, the height of clusters and bundles was determined by a height histogram (Figure 3b) across the selected area, marked in Figure 3a. The peak distance was used as a measure for the average height. The width and length of the cylindrical bundles was estimated from a number of height profiles to have an impression on the size. The scales of the images are either given via scale bars or the length of the whole image is noted.

\section{Results and Discussion}

\section{$\underline{\text { Size and Structure of Dispersion Clusters and Sub-Monolayer Films }}$}

As a main analysis method, we determined the size of clusters by AFM after deposition on flat substrates. In Figure 4a,b, Nafion clusters from hydro-alcoholic (Figure 4a) and from water-based (Figure 4b) dispersion were placed on a flat mica surface by drop-casting with a low ionomer concentration. For AFM measurements areas with a small coverage were examined. The clusters originating from the alcoholic dispersion were more spherical with a width of roughly $10-30 \mathrm{~nm}$ and a length of roughly 30-100 $\mathrm{nm}$. The majority of clusters originating from the water-based dispersion were more cylindrical and these bundles had a width of approximately $10 \mathrm{~nm}$ and a length of approximately 50-100 nm. Mostly fibrillary, rod-like bundles were reported for Nafion. The sizes derived by AFM are in agreement with the reported values, i.e from Ngo et al. 
and Welch et al. $(5,21)$. Loppinet et al. found smaller rods with only $2 \mathrm{~nm}$ radius by small angle neutron spectroscopy (SANS) (22).

Although the sizes measured by AFM fit to most of the literature data, due to convolution of the AFM tip with the polymer, the width (but not the height) of the bundles appears larger. To minimize this influence, ultra-sharp AFM tips were used in addition. Furthermore, a larger size measured by AFM may result from an additional clustering on the surface during solvent evaporation.

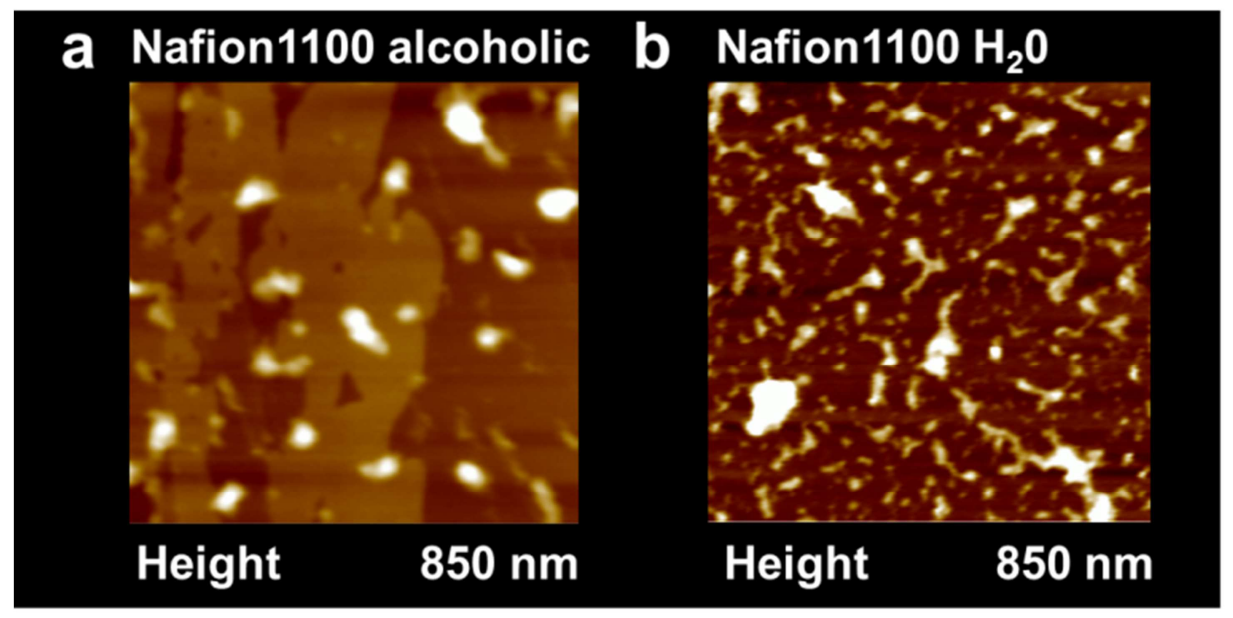

Figure 4. AFM height images of Nafion 1100, drop-cast on graphene sheets, (a) from hydro-alcoholic dispersion, clusters of 10-30 nm x 30-100 nm, (b) from water-based dispersion, cylindrical bundles of $10 \mathrm{~nm} \times 50-100 \mathrm{~nm}$; the lengths refer to the total image size.

\section{Thickness of Sub-Monolayer Films}

In Figure 5, a sub-monolayer film and multilayers deposited on mica from waterbased Aquivion D79-25BS dispersion are presented. The formation of multilayers is visible in the topography image of Figure 5a. The first step height was $2.1 \mathrm{~nm}$ high at the dense edge. At the terrace, large holes down to the substrate are present and the film height measured $2.6 \mathrm{~nm}$. The second layer had a thickness of $2.0 \mathrm{~nm}$, the step to the third layer was $2 \mathrm{~nm}$. The layer morphology is different. The higher-resolution image of the first layer (Figure 5b) shows the dense edge without holes, the thinner films indicate a decreased water layer directly at the edge. With increasing distance from the edge, many large holes are present. The structure of the second layer (Figure 5c) is different and consists of more isolated cylindrical bundles that form a connected network. No holes down to the substrate were detected any more. The third layer has a dense granular surface. An area on the substrate with low and high cluster density in one layer is shown in Figure 5d. 


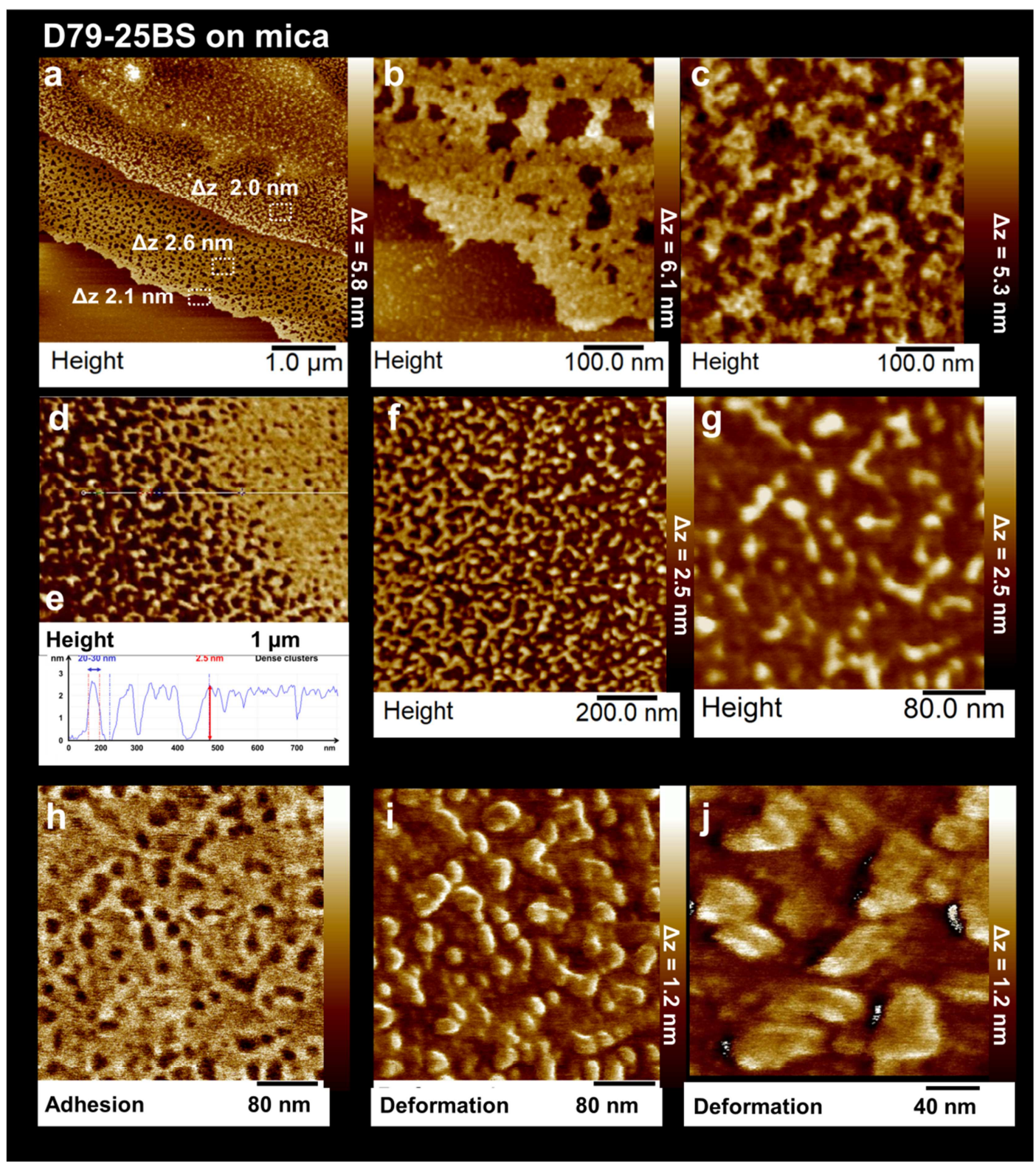

Figure 5. AFM images of drop-cast water-based Aquivion D79-25BS dispersion on mica (a) topography image of position with 4 layers, (b) high-resolution image of first layer with dense edge and $2.1 \mathrm{~nm}$ step height at edge and $2.6 \mathrm{~nm}$ within terrace, (c) highresolution image of the second layer with $2.0 \mathrm{~nm}$ step height within terrace, (d) topography image with different ionomer density in one layer, (e) height profile along the line in (d), (f) topography image of the area covered with isolated bundles, (g) highresolution topography image with individual Aquivion clusters of $2.5 \mathrm{~nm}$ height, (h) corresponding adhesion force mapping of $(\mathrm{g})$ with low adhesive cylindrical bundles of 20 x $44 \mathrm{~nm}$, (i) corresponding deformation mapping of (g), and (j) zoom into the deformation mapping with internal higher deformable zig-zack and lamellar lines with $\approx$ $5 \mathrm{~nm}$ width.

In Figure 5e the height profile along the line of Figure $5 \mathrm{~d}$ is presented. The isolated and the densely packed bundles have the same height of $2.5 \mathrm{~nm}$ and represent a single ionomer layer. The densely packed area still has many pin-holes. The isolated bundles 
have a width of approximately $20-30 \mathrm{~nm}$. The higher resolution topography image in Figure 5f,g exhibits a progressive network formation of the cylindrical bundles. These bundles have a granular structure along their length (Figure $5 \mathrm{~g}$ ). A comparison of the corresponding topography (Figure $5 \mathrm{~g}$ ), adhesion (Figure $5 \mathrm{~h}$ ), and deformation (Figure 5i) mappings of the same area exhibits a different structure of the granules depending on the measured property. In the adhesion mapping (Figure 5h), the dark isolated granules along the cylinders are indicative of ionic domains and have a size of roughly $20 \mathrm{~nm} \times 44 \mathrm{~nm}$. The deformation mappings of these areas show an internal structure (Figure 5i). The high-resolution stiffness mapping (Figure $5 \mathrm{j}$ ) exhibits highly deformable, zick-zack or lamellar lines that can be assumed as positions of backbone bundles with an AFMmeasured size of $\approx 5 \mathrm{~nm}$.

\section{$\underline{\text { Ionomer on Different Substrates }}$}

A comparison of the polymer structure on substrates with different surface energy is presented in Figure 6. On hydrophilic mica and $\mathrm{Si}$, the sulfonic acid groups that terminate the side chains and form the ionic phase are attached to the water layer at the substrate; on graphene the ionomer backbone chains are directly attached to the hydrophobic surface $(15,16)$. Without the underlying water layer, the layer height is smaller. Figure 6a exhibits the topography image of one bundle of water-based D83-25BS dispersion on hydrophilic Si. The polymer bundle measures more than 1 micrometer in length. The bundle exhibits a granular structure along its chain. In the adhesion force mapping (Figure 6b), these grains are of ionic nature according to their low-adhesion. The polymer is curved around a flat, $2 \mathrm{~nm}$-high island with extremely high adhesion, most-likely covered with a water layer attached to the ionic clusters of the polymer. A statistical evaluation of D83-25BS bundle height on $\mathrm{Si}$ is shown in Figure $7 \mathrm{~b}$.

The same ionomer deposited on a graphene surface has formed highly branched polymer bundles. A high-resolution deformation image is given in Figure 6c. The polymer bundle has a width of $20 \mathrm{~nm}$ and a height of $1.5 \mathrm{~nm}$. On the hydrophobic substrate, the ionic domains are most-likely oriented upwards to the bundle top and are not stretched to both sides of the bundle. From measurements of the step heights on Aquivion membranes, a corresponding step height was determined. It relates to the size of the main chain and the length of the half-stretched side chains (29). The bundle in Figure $6 \mathrm{~b}$ is formed of two parallel structures decorated with highly deformable double spots, most-likely formed by the two side-chains oriented to the top; also the height of the polymer bundles is smaller than on mica and Si.

On hydrophilic substrates such as mica or $\mathrm{Si}$, the ionic domains formed by the side chains are assumed to be attached to the substrate and are oriented to both sides of the polymer bundle resulting in a bundle thickness of $2.5 \mathrm{~nm}$. This explains the larger bundle width on mica and $\mathrm{Si}$ compared to graphene. The absence of a water layer below the polymer is the cause of the smaller height on graphene. The height of small ionomer layers that are formed along steps of the graphene edges (Figure 6d) also measures 1.5 $\mathrm{nm}$; the ionomer dots on these small films represent the initial growth of a second layer with a total height of $2.3 \mathrm{~nm}$. 

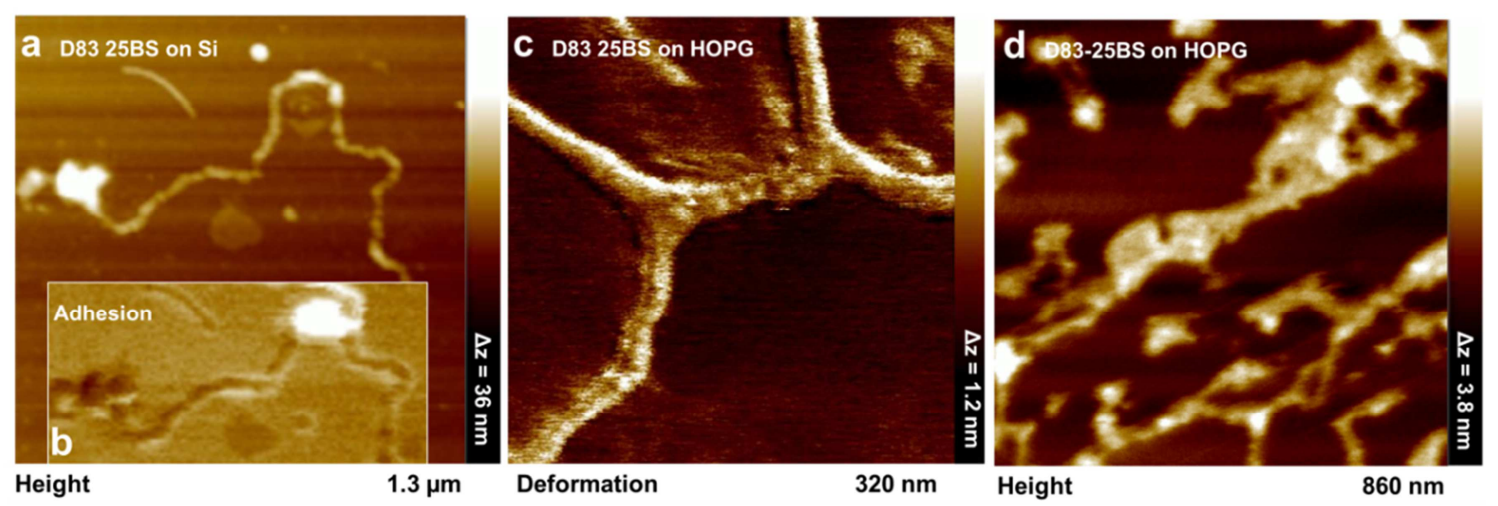

Figure 6. AFM topography image of water-based D83-25BS dispersion with a concentration of 0.0025 wt.\% of ionomer, (a) on $\mathrm{Si}$ with a bundle length of approximately $1 \mu \mathrm{m}$, (b) corresponding adhesion force mapping of (a), (c) highresolution AFM deformation mapping on graphene with bundles of $20 \mathrm{~nm}$ width and 1.5 $\mathrm{nm}$ height, and (d) area with small layers on graphene with $1.5 \mathrm{~nm}$-thick first layer and an initial second layer of ionomer spots with a total height of $2.3 \mathrm{~nm}$; the lengths refer to the total image size.

A comparison of the resulting Aquivion D83 film structure on Pt and Si by the same drop-cast coating leads to completely different coverage. On Pt, a closed film with a thickness of $8 \mathrm{~nm}$ was formed at the center of the drop (Figure 7a), whereas on $\mathrm{Si}$, a loose network of $2.5 \mathrm{~nm}$-thick cylindrical bundles covers $41 \%$ of the surface (Figure $7 \mathrm{~b}$ ). This significant difference emphasizes the importance of the local surface energy for ionomer film formation.

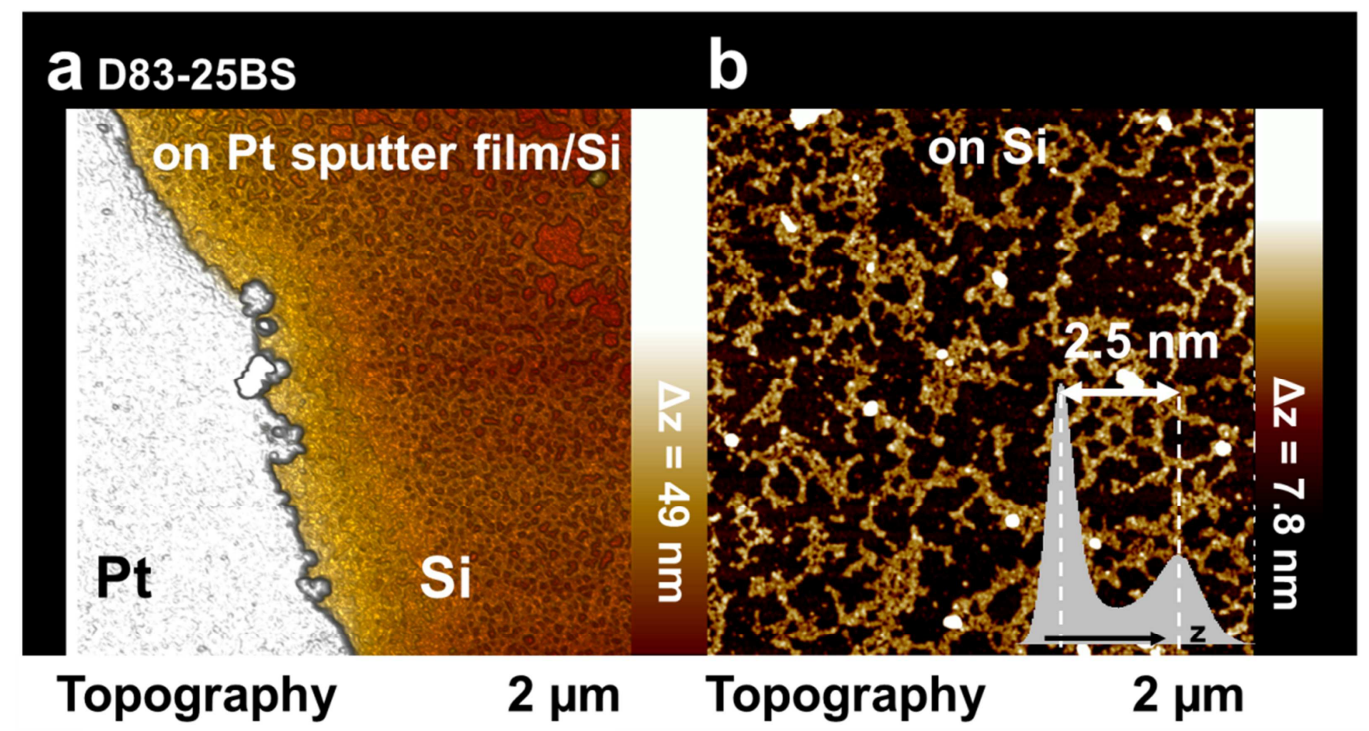

Figure 7. (a) AFM topography image of drop-cast, water-based D83-25BS dispersion with a concentration of 0.005 wt.\%, (a) simultaneously drop-casted, half-Pt coated $\mathrm{Si}$ substrate, close to drop center, measured at $50 \% \mathrm{RH}$, and (b) network-like ionomer coverage on Si side at right with $2.5 \mathrm{~nm}$ height of bundles (height histogram in the inset); the lengths refer to the total image size. 


\section{$\underline{\text { Comparison of Coating Methods }}$}

Depending on the position within the drop the local ionomer concentration can vary and a loose ionomer coverage with isolated clusters (Figure 4 and 5) or the formation of layers (Figure 5d) or multilayers (Figure 5a) was observed. Furthermore, the cluster structure depends on the solvent, as the size of dispersion clusters is different (Figure 4). A major influence on the orientation of bundles and the film thickness results from the substrate surface energy (Figure 6). Even in case of two hydrophilic substrates as Pt and $\mathrm{Si}$, the coverage is influenced by i.e. the surface roughness (Figure 7). The minimum layer thickness of dense Nafion and Aquivion films depends on humidity and substrate surface energy and is $\approx 4 \mathrm{~nm}$ for Nafion and due to the shorter side-chains $\approx 3.6 \mathrm{~nm}$ for Aquivion $(30,31)$. This thickness was also found by modeling of humid Nafion on substrates with different surface energy $(15,16)$. The thickness that results from coating by self-assembling strongly depends on the ionomer concentration and only slightly on the emersion time (32).

From the experiments it can be derived that depending on the deposition procedure, either layers of $1.5 \mathrm{~nm}$ or $2.5 \mathrm{~nm}$ for hydrophobic or hydrophilic substrates, respectively, or dense ionomer films with a minimum thickness of approximately $4 \mathrm{~nm}$ are formed. During self-assembling in a large volume, the ionomer concentration in the dispersion stays constant during deposition. A continued interaction of already deposited ionomer and dispersion particles finally leads to an equilibrium in the resulting film morphology, density, and thickness. In this situation the minimum film thickness observed is $4 \mathrm{~nm}$, a higher concentration leads to thicker films.

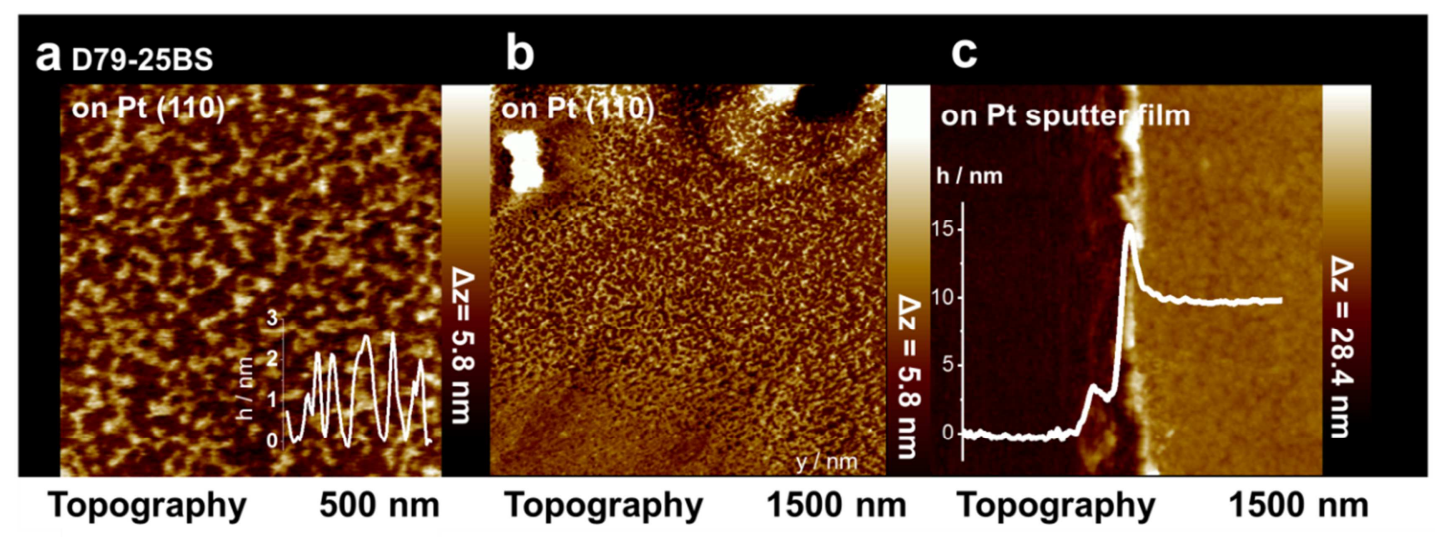

Figure 8. AFM topography images of water-based D79-25BS, (a) drop-cast from 0.005 wt.\% ionomer dispersion on a Pt(110) single crystal surface, measured at RH $50 \%$, a profile line across the bundles overlaid, (b) larger area of the same sample as shown in (a), and (c) dense $10 \mathrm{~nm}$-thick film from $0.25 \mathrm{wt} \%$ ionomer dispersion after selfassembly for $2 \mathrm{~h}$ on a Pt sputter layer, measured at $50 \% \mathrm{RH}$; the lengths refer to the total image size.

The difference in resulting ionomer layer by drop-cast and self-assembling is shown in Figure 8. In Figure 8a,b, a drop-cast ionomer coating of water-based D79-25BS from 0.005 wt. $\%$ on a $\operatorname{Pt}(110)$ single-crystal surface is given after $1 \mathrm{~h}$ of evaporation. The crystal was chosen as substrate due to its low surface roughness. Distinct cylindrical 
bundles with a height of $2.3 \mathrm{~nm}$, derived from the overlaid profile line, were formed. In Figure $8 \mathrm{c}$, the ionomer coverage after $2 \mathrm{~h}$ of self-assembling in the same ionomer dispersion with a concentration of $0.25 \mathrm{wt} . \%$ resulted in a dense film with a thickness of $10 \mathrm{~nm}$ as visible in the overlaid profile line across the edge (Figure $8 \mathrm{c}$ ).

In a deposited drop of dilute dispersion, the situation is different. Due to the solvent evaporation during drop casting, the initial ionomer concentration increases and local differences at the edges and the center of the substrate occur. Due to the low total amount, after some time all clusters in the liquid are deposited on the surface and no further growth or structure change of the initially deposited bundles occurs. In this case the layers consist of more or less loosely connected single bundle-height layers or loose networks.

\section{Consequences for Ionomer Film Formation in Electrodes}

In a typical ink for fuel cell electrode preparation the concentration of ionomer is in the range of 0.1-4 wt.\%. After ink preparation, self-assembling of the ionomer on the $\mathrm{Pt} / \mathrm{C}$ particles is taking place at constant high ionomer concentration in the dispersion. Under this condition, the formation of dense layers with a thickness of at least $4 \mathrm{~nm}$ can be assumed. It was observed in the experiments that the first $4 \mathrm{~nm}$ thick layer attached to the substrate cannot be easily removed, i.e. by blowing with argon, whereas thicker residuals could be removed by this procedure. From the self-assembling experiments one would expect that the ionomer film thickness that depends on the ionomer concentration in the ink would be much larger. Following the results of Paul et al., for a concentration of $5 \mathrm{wt} . \%$ of Nafion a thickness of up to $300 \mathrm{~nm}$ was measured. Therefore, additional factors may influence the final thickness in the electrode. One possibility is a removal of the topmost material due to the coating method such as spraying; another influence is the final MEA processing. Most of the MEAs are hot-pressed after preparation. The heating above the glass temperature under high pressure most-likely further changes the ionomer films within the electrodes. It can be assumed that under these conditions of high temperature and pressure a lamellar ionomer structure is favored over a cluster-like. Also in this case, the topmost fraction of the soft thicker ionomer films may be squeezed out to open pores and may form ionomer agglomerates resulting in thin remaining ionomer films around the agglomerates. This process needs be further studied.

\section{Conclusions}

The formation of sub-monolayer and nanothin ionomer films from Nafion and Aquivion PFSA dispersions was studied to get insight into the formation of ionomer films in fuel cell electrodes. The ionomer was deposited on Pt, single crystal and sputter layers, and carbon, materials used in electrodes, and on flat hydrophilic Si and mica. By drop-casting of dilute dispersions, isolated dispersion clusters were observed on flat substrates and the size and morphology were analyzed by material-sensitive AFM. In case of water-based dispersions, the mostly cylindrical bundles were formed. They consisted of smaller spherical clusters, identified by adhesion force mapping as ionic domains. The resulting ionomer coverage was dependent on the coating method. Due to a locally different ionomer concentration during evaporation and a fast depletion of the ionomer in the solvent, different ionomer coverage occurred on the samples. At positions 
with higher concentration within the drop, a connected network of the bundles and also multilayers of up to 4 layers were formed. The height and width of the bundles and their branching depended on the substrate energy. At comparable humidity, on hydrophobic graphene bundles and layers of $1.5 \mathrm{~nm}$-thick were formed; on the hydrophilic substrates, the thickness was $2.5 \mathrm{~nm}$. Dense ionomer films were received by self-assembling from a constant ionomer concentration; after sufficient time the formation of at least $4 \mathrm{~nm}$-thick films occurred. It is assumed that in an ink for electrode preparation a similar process takes place and for typical ionomer concentrations of 0.1-0.3 wt.\%, the ionomer deposition on Pt/C particles immediately starts and ionomer films of $4 \mathrm{~nm}$ and thicker are formed. The final structure of the ionomer films in the electrodes is assumed to be further changed by a final hot-pressing step that is assumed to lead to thinner lamellar films.

\section{Acknowledgments}

The research leading to these results received funding from the European Union's Seventh Framework Programme (FP7/2007-2013) for the Fuel Cells and Hydrogen Joint Technology Initiative under grant agreement $n^{\circ}$ 621237, "INSIDE- In-situ Diagnostics in Water Electrolyzers".

\section{References}

1. S. Holdcroft, Chem. Mater., 26, 381-393 (2014).

2. M. K. Debe et al., J. Electrochem. Soc., 159, K165-K176 (2012).

3. K. Karan, Curr. Opin. Electrochem., 5, 27-35 (2017).

4. T. Morawietz, M. Handl, C. Oldani, K. A. Friedrich, and R. Hiesgen, ACS Appl. Mater. Interfaces, 8, 27044-27054 (2016).

5. T. T. Ngo, T. L. Yu, and H.-L. Lin, J. Power Sources, 225, 293-303 (2013).

6. A. P. Young, J. Stumper, S. Knights, and E. Gyenge, J. Electrochem. Soc., 157, B425 (2010).

7. T.-H. Kim, J.-Y. Yi, C.-Y. Jung, E. Jeong, and S.-C. Yi, Int. J. Hydrog. Energy, 42, 478-485 (2017).

8. Handl, M., Morawietz, T., Friedrich, K. A., Hiesgen, R., ECST Seattle (2018).

9. M. Eikerling and A. A. Kulikovsky, Polymer electrolyte fuel cells: physical principles of materials and operation, p. 541, CRC Press, Taylor \& Francis Group, CRC Press is an imprint of the Taylor \& Francis Group, an informa business, Boca Raton, (2015).

10. A. Kongkanand and M. F. Mathias, J. Phys. Chem. Lett., 7, 1127-1137 (2016).

11. T. Muzaffar, T. Kadyk, and M. Eikerling, Sustain. Energy Fuels (2018) http://pubs.rsc.org/en/Content/ArticleLanding/2018/SE/C8SE00026C.

12. T. Morawietz, M. Handl, C. Oldani, K. A. Friedrich, and R. Hiesgen, Fuel Cells (2018) http://doi.wiley.com/10.1002/fuce.201700113.

13. R. Hiesgen, T. Morawietz, M. Handl, M. Corasaniti, and K. A. Friedrich, Electrochimica Acta, 162, 86-99 (2015).

14. M. Handl, Morawietz, T., Friedrich, Hiesgen, R., ECS Trans., 85, 889-903 (2018).

15. D. Damasceno Borges, A. A. Franco, K. Malek, G. Gebel, and S. Mossa, $A C S$ Nano, 7, 6767-6773 (2013). 
16. D. Damasceno Borges, G. Gebel, A. A. Franco, K. Malek, and S. Mossa, J. Phys. Chem. C, 119, 1201-1216 (2014).

17. D. Paul, A. Fraser, J. Pearce, and K. Karan, in ECS Transactions, vol. 41, p. 1393-1406 (2011) http://ecst.ecsdl.org/cgi/doi/10.1149/1.3635670.

18. A. Kusoglu and A. Z. Weber, Chem. Rev., 117, 987-1104 (2017).

19. P. Aldebert et al., J. Phys., 49, 2101-2109 (1988).

20. E. Szajdzinska-Pietek, S. Schlick, and A. Plonka, Langmuir, 10, 2188-2196 (1994).

21. C. Welch et al., ACS Macro Lett., 1, 1403-1407 (2012).

22. B. Loppinet and G. Gebel, Langmuir, 14, 1977-1983 (1998).

23. B. Loppinet, G. Gebel, and C. E. Williams, J. Phys. Chem. B, 101, 1884-1892 (1997).

24. M. Ghelichi, K. Malek, and M. H. Eikerling, Macromolecules, 49, 1479-1489 (2016).

25. R. Hiesgen, Morawietz, T., Friedrich., Membranes, 2, 783-803 (2012).

26. R. Hiesgen, Morawietz, T., Friedrich, Electrochimica Acta, 110, 292-305 (2013).

27. R. Hiesgen, T. Morawietz, M. Handl, M. Corasaniti, and K. A. Friedrich, Electrochimica Acta, 162, 86-99 (2015).

28. D. K. Paul, K. Karan, A. Docoslis, J. B. Giorgi, and J. Pearce, Macromolecules, 46, 3461-3475 (2013).

29. R. Hiesgen et al., Membranes, 2, 783-803 (2012).

30. Devproshad Paul, A. Fraser, J. Devproshad, and K. Karan, ECS Trans., 41, 13931406 (2011).

31. D. K. Paul, A. Fraser, and K. Karan, Electrochem. Commun., 13, 774-777 (2011).

32. D. Paul, A. Fraser, J. Pearce, and K. Karan, in ECS Transactions,, vol. 41, p. 1393-1406 (2011). 